PROCEEDINGS OF THE

AMERICAN MATHEMATICAL SOCIETY

Volume 134, Number 7 , Pages 2015-2018

S 0002-9939(05)08413-3

Article electronically published on December 19, 2005

\title{
UNIQUE CONTINUATION FOR THE SYSTEM OF ELASTICITY IN THE PLANE
}

\author{
L. ESCAURIAZA
}

(Communicated by David S. Tartakoff)

\begin{abstract}
We prove the strong unique continuation property for the Lamé system of elastostatics in the plane, $\nabla \cdot\left(\mu\left(\nabla u+\nabla u^{t}\right)\right)+\nabla(\lambda \nabla \cdot u)=0$, with variable Lamé coefficients $\mu, \lambda$, when $\mu$ is Lipschitz and $\lambda$ is measurable.
\end{abstract}

\section{INTRODUCTION}

The main purpose of this note is to study the strong unique continuation property for the Lamé system of linearized elastostatics in the plane

$$
\nabla \cdot\left(\mu(x)\left(\nabla u+\nabla u^{t}\right)\right)+\nabla(\lambda(x) \nabla \cdot u)=0 .
$$

The functions $\mu$ and $\lambda$ are known as the Lamé coefficients, $\nabla u$ is the $2 \times 2$ matrix $\left(\partial_{i} u^{j}\right)_{i, j=1}^{2}$ and $\nabla u^{t}$ its transpose $\left(\partial_{j} u^{i}\right)_{i, j=1}^{2}$. The system (1.1) is elliptic when $\mu$ and $\lambda$ are in $L^{\infty}\left(\mathbb{R}^{2}\right)$ and for some $\kappa>0$,

$$
\mu(x) \geq \kappa \text { and } 2 \mu(x)+\lambda(x) \geq \kappa, \text { when } x \in \mathbb{R}^{2} .
$$

The previous results in the literature are the following: Dehman and Robbiano proved the weak unique continuation property for the system of elastostatics in $\mathbb{R}^{n}$, $n \geq 2$, when $\lambda, \mu \in \mathcal{C}^{\infty}\left(\mathbb{R}^{n}\right)\left[6\right.$, Ang, Ikehata, Trong and Yamamoto for $\lambda \in \mathcal{C}^{2}\left(R^{n}\right)$ and $\mu \in \mathcal{C}^{3}\left(\mathbb{R}^{n}\right)$ 1], Weck for $\lambda, \mu \in \mathcal{C}^{2}\left(\mathbb{R}^{n}\right)(11$, [12), and Nakamura and Wang for the Lamé system with residual stress for $\lambda, \mu \in \mathcal{C}^{1,1}\left(\mathbb{R}^{n}\right)$ [10. The strong unique continuation property has been proved in $\mathbb{R}^{3}$ by C. Lin for twice differentiable Lamé coefficients [8], by Alessandrini and Morasi in $\mathbb{R}^{n}, n \geq 2$, for $\lambda, \mu \in \mathcal{C}^{1,1}\left(\mathbb{R}^{n}\right)$, and by C.L. Lin and J.N. Wang, when $\lambda, \mu$ are Lipschitz functions in $\mathbb{R}^{2}[9$. In this note we prove the following result:

Theorem 1. Assume that $u=\left(u_{1}, u_{2}\right) \in W^{1,2}(B)$ satisfies in the sense of distributions (1.1) in the unit ball $B$ of the plane, $\mu$ is a Lipschitz function, $\lambda$ is measurable, and for all $k \geq 1$ there is some constant $C_{k}$ such that

$$
\int_{B_{r}}|u|^{2} d x \leq C_{k} r^{k}, \text { when } 0<r \leq 1 .
$$

Then, $u \equiv 0$ in $B$.

Received by the editors December 7, 2004 and, in revised form, February 9, 2005.

2000 Mathematics Subject Classification. Primary 35J45; Secondary 35B60.

Key words and phrases. Unique continuation, elasticity.

The author was supported by MEC grant MTM2004-03029 and by the European Commission via the network Harmonic Analysis and Related Problems, project number RTN2-2001-00315.

(C)2005 American Mathematical Society Reverts to public domain 28 years from publication 
The same result holds when we add to the system of elasticity (1.1) lower order terms with bounded measurable coefficients.

Scalar elliptic operators in the plane with measurable coefficients, both in divergence or non-divergence form, have the strong unique continuation property and perhaps the same holds for the system (1.1) when $\lambda$ and $\mu$ are measurable. Scalar elliptic operators in the plane in non-divergence form have the strong unique continuation property because its solutions $u$ satisfy, $u_{x}-i u_{y}=f \circ \aleph$, where $f$ is some analytic function and $\aleph$ is a certain quasi-conformal homeomorphism of the plane, which verifies

$$
N^{-1}|z-w|^{\alpha} \leq|\aleph(z)-\aleph(w)| \leq N|z-w|^{\beta} \text {, when } z, w \in \mathbb{R}^{2},
$$

for some positive constants $N, \alpha$ and $\beta$ ([4, [5]). In the divergence case, $u=$ $\Re(f \circ \aleph)$, where $f$ and $\aleph$ are as above 3 .

Our improvement of the result by C.L. Lin and J.N. Wang in 9 closely follows their arguments. In the next section the reader will realize that the difference between the two arguments is a simple algebraic calculation generalizing the following fact:

Assume that $\mu$ is a constant, $\lambda$ is measurable, and $u=\left(u_{1}, u_{2}\right)$ is a solution of (1.1) in $B$. Then, $f(z)=\mu\left(\partial_{1} u_{2}-\partial_{2} u^{1}\right)-i(2 \mu+\lambda)\left(\partial_{1} u_{1}+\partial_{2} u_{2}\right)$ is an analytic function in $B$.

Here and in the sequel we use the following notation: $\left(x_{1}, x_{2}\right)$ denotes a point in the plane, $\partial_{1}=\frac{\partial}{\partial x_{1}}$, and $\partial_{2}=\frac{\partial}{\partial x_{2}}$.

\section{An algebraic CALCUlation}

Proof of Theorem 1. The Lamé equations in the plane are

$$
\begin{aligned}
& 2 \partial_{1}\left(\mu \partial_{1} u_{1}\right)+\partial_{2}\left(\mu\left(\partial_{1} u_{2}+\partial_{2} u_{1}\right)\right)+\partial_{1}\left(\lambda\left(\partial_{1} u_{1}+\partial_{2} u_{2}\right)\right)=0, \\
& \partial_{1}\left(\mu\left(\partial_{1} u_{2}+\partial_{2} u_{1}\right)\right)+2 \partial_{2}\left(\mu \partial_{2} u_{2}\right)+\partial_{2}\left(\lambda\left(\partial_{1} u_{1}+\partial_{2} u_{2}\right)\right)=0,
\end{aligned}
$$

and can be rewritten as

$$
\begin{aligned}
& \partial_{2}\left(\mu\left(\partial_{1} u_{2}+\partial_{2} u_{1}\right)\right)-2 \partial_{1}\left(\mu \partial_{2} u_{2}\right)+\partial_{1}\left((2 \mu+\lambda)\left(\partial_{1} u_{1}+\partial_{2} u_{2}\right)\right)=0, \\
& \partial_{1}\left(\mu\left(\partial_{1} u_{2}+\partial_{2} u_{1}\right)\right)-2 \partial_{2}\left(\mu \partial_{1} u_{1}\right)+\partial_{2}\left((2 \mu+\lambda)\left(\partial_{1} u_{1}+\partial_{2} u_{2}\right)\right)=0 .
\end{aligned}
$$

On the other hand,

$$
\begin{aligned}
& -2 \partial_{1}\left(\mu \partial_{2} u_{2}\right)=-2 \partial_{2}\left(\mu \partial_{1} u_{2}\right)+2 \partial_{2} \mu \partial_{1} u_{2}-2 \partial_{1} \mu \partial_{2} u_{2}, \\
& -2 \partial_{2}\left(\mu \partial_{1} u_{1}\right)=-2 \partial_{1}\left(\mu \partial_{2} u_{1}\right)+2 \partial_{1} \mu \partial_{2} u_{1}-2 \partial_{2} \mu \partial_{1} u_{1} .
\end{aligned}
$$

Plugging these formulas in (2.2) we get the following system of equations:

$$
\begin{array}{r}
-\partial_{2}\left(\mu\left(\partial_{1} u_{2}-\partial_{2} u_{1}\right)\right)+\partial_{1}\left((2 \mu+\lambda)\left(\partial_{1} u_{1}+\partial_{2} u_{2}\right)\right)+2 \partial_{2} \mu \partial_{1} u_{2}-2 \partial_{1} \mu \partial_{2} u_{2}=0 \\
\partial_{1}\left(\mu\left(\partial_{1} u_{2}-\partial_{2} u_{1}\right)\right)+\partial_{2}\left((2 \mu+\lambda)\left(\partial_{1} u_{1}+\partial_{2} u_{2}\right)\right)+2 \partial_{1} \mu \partial_{2} u_{1}-2 \partial_{2} \mu \partial_{1} u_{1}=0
\end{array}
$$

Setting $v_{1}=u_{1}, v_{2}=u_{2}, v_{3}=\mu\left(\partial_{1} u_{2}-\partial_{2} u_{1}\right)$ and $v_{4}=(2 \mu+\lambda)\left(\partial_{1} u_{1}+\partial_{2} u_{2}\right)$, it follows from (2.3) and the formulas

$$
\partial_{1} u_{2}=\frac{v_{3}}{\mu}+\partial_{2} v_{1} \quad, \quad \partial_{1} u_{1}=\frac{v_{4}}{2 \mu+\lambda}-\partial_{2} v_{2},
$$


that $v_{1}, v_{2}, v_{3}$ and $v_{4}$ satisfy the system of equations

$$
\left\{\begin{array}{l}
\partial_{1} v_{1}+\partial_{2} v_{2}-\frac{v_{4}}{2 \mu+\lambda}=0 \\
\partial_{1} v_{2}-\partial_{2} v_{1}-\frac{v_{3}}{\mu}=0 \\
\partial_{1} v_{3}+\partial_{2} v_{4}+2 \partial_{1} \mu \partial_{2} v_{1}+2 \partial_{2} \mu \partial_{2} v_{2}-\frac{2 \partial_{2} \mu}{2 \mu+\lambda} v_{4}=0 \\
\partial_{1} v_{4}-\partial_{2} v_{3}+2 \partial_{2} \mu \partial_{2} v_{1}-2 \partial_{1} \mu \partial_{2} v_{2}+\frac{2 \partial_{2} \mu}{\mu} v_{3}=0
\end{array}\right.
$$

which can be written in matrix form as

$$
\partial_{1} V+J \partial_{2} V+M V=0
$$

where $V=\left(v_{1}, v_{2}, v_{3}, v_{4}\right)^{t}$ and $J, M$ are the $4 \times 4$ matrices

$$
J=\left(\begin{array}{cccc}
0 & 1 & 0 & 0 \\
-1 & 0 & 0 & 0 \\
2 \partial_{1} \mu & 2 \partial_{2} \mu & 0 & 1 \\
2 \partial_{2} \mu & -2 \partial_{1} \mu & -1 & 0
\end{array}\right) \quad, \quad M=\left(\begin{array}{cccc}
0 & 0 & 0 & -\frac{1}{2 \mu+\lambda} \\
0 & 0 & -\frac{1}{\mu} & 0 \\
0 & 0 & 0 & -\frac{2 \partial_{2} \mu}{2 \mu+\lambda} \\
0 & 0 & \frac{2 \partial_{2} \mu}{\mu} & 0
\end{array}\right)
$$

The Cauchy-Riemann equations and (2.3) show that the claim at the end of the Introduction does hold.

If $u=\left(u_{1}, u_{2}\right) \in W^{1,2}(B)$ is a solution in the sense of distributions of (1.1), the last two identities in (2.5) give

$$
\partial_{\bar{z}}\left(v_{3}-i v_{4}\right)=f \text { in } B,
$$

where $\partial_{\bar{z}}=\frac{1}{2}\left(\partial_{1}+i \partial_{2}\right)$ is the Cauchy-Riemann operator and $|f| \leq N|\nabla u|$ for some $N>0$ depending on the constant $\kappa$ in (1.2) and $\|\nabla \mu\|_{L^{\infty}\left(\mathbb{R}^{2}\right)}$. The gradient of the inverse of the Cauchy-Riemann operator is a Calderón-Zygmund operator, and so (2.7) and standard elliptic regularity implies

$$
\int_{B_{r}}\left|\nabla v_{3}\right|^{2}+\left|\nabla v_{4}\right|^{2} d x \leq \frac{N}{r^{2}} \int_{B_{2 r}}|\nabla u|^{2} d x, \text { when } 0<r \leq 1 / 2 .
$$

This argument, the Cacciopoli-type inequality

$$
\int_{B_{r}}\left|\nabla u+\nabla u^{t}\right|^{2} d x \leq \frac{N}{r^{2}} \int_{B_{2 r}}|u|^{2} d x, \text { when } 0<r \leq 1 / 2,
$$

and Korn's inequality [7]

$$
2 \int_{B}|\nabla \varphi|^{2} d x \leq \int_{B}\left|\nabla \varphi+\nabla \varphi^{t}\right|^{2} d x, \text { when } \varphi=\left(\varphi_{1}, \varphi_{2}\right) \in W_{0}^{1,2}(B),
$$

imply that when $u=\left(u_{1}, u_{2}\right) \in W^{1,2}(B)$ satisfies the conditions in Theorem 1 the following holds: $V \in W_{l o c}^{1,2}(B), V$ verifies pointwise almost everywhere the system (2.6) and has a zero of infinite order at the origin i.e., for each $k \geq 1$ there is some constant $C_{k}$ such that

$$
\int_{B_{r}}|V|^{2} d x \leq C_{k} r^{k}, \text { when } 0<r \leq 1 .
$$

At this point we recall the following result which proves Theorem 1, The interested reader will find its proof between the lines in 9 . 
Theorem 2. Assume that $V \in W_{l o c}^{1,2}(B), V=\left(v_{1}, v_{2}, v_{3}, v_{4}\right)^{t}$ is a solution in the unit ball $B \subset \mathbb{R}^{2}$ of a first order system of the form (2.6), where the $4 \times 4$ matrices $J$ and $M$ verify $J, M \in L^{\infty}\left(\mathbb{R}^{2}\right)$, and

$$
J=\left(\begin{array}{cccc}
0 & 1 & 0 & 0 \\
-1 & 0 & 0 & 0 \\
\alpha & \beta & 0 & 1 \\
\gamma & \delta & -1 & 0
\end{array}\right)
$$

for some measurable functions $\alpha, \beta, \gamma$ and $\delta$. Then, if $V$ has zero of infinite order at the origin, $V \equiv 0$ in $B$.

The claim after Theorem 1 follows in the same way from Theorem 2 , the corresponding analog of (2.2), and the formulas (2.4).

As stated in the Introduction, This proof only differs from the arguments in 9 on an algebraic calculation. The arguments in 9] do not imply Theorem 1 because they choose as the components of $V$ the functions, $v_{1}=u_{1}, v_{2}=u_{2}$, $v_{3}=\partial_{1} u_{2}-\partial_{2} u_{1}$ and $v_{4}=\partial_{1} u_{1}+\partial_{2} u_{2}$. With these choices, $V$ is a solution of an elliptic system of the type (2.6) but with matrices $J$ and $M$, which depend on $\nabla \mu$ and $\nabla \lambda$.

\section{REFERENCES}

[1] D.D. Ang, M. Ikehata, D.D. Trong, and M. Yamamoto, Unique continuation for a stationary isotropic Lamé system with variable coefficients, Comm. in PDE 23 (1998), 371-385. MR:1608540 (98j:35049)

[2] G. Alessandrini and R. Magnanini, Elliptic equations in divergence form, geometric critical points of solutions and Stekloff eigenfunctions, SIAM J. Math. Anal. 5 (1994), 1259-1268. MR:1289138 (95f:35180)

[3] G. Alessandrini and A. Morassi, Strong unique continuation for the Lamé system of elasticity, Comm. in PDE 26 (2001), 1787-1810. MR.1865945 (2003j:35030)

[4] L. Bers, F. John, and M. Schechter, Partial Differential Equations, Interscience, New York, 1964. MR0163043 (29:346)

[5] L. Bers and L. Nirenberg, On a representation theorem for linear elliptic systems with discontinuous coefficients and its applications, In: Convegno Internazionale sulle Equazioni alle Derivate Parziali, Cremonese, Roma (1955), 111-138. MR0076981 (17:974d)

[6] B. Dehman and L. Robbiano, La propiété du prolongement unique pour un système elliptique: le système Lamé, J. Math. Pures Appl. 72 (1993), 475-492. MR1239100 (94h:35051)

[7] K.O. Friedrichs, On the boundary value problems of the theory of elasticity and Korn's inequality, Annals of Math. 48 (1947), 441-471. MR0022750 (9:255b)

[8] C.L. Lin, Strong unique continuation for an elasticity system with residual stress, Indiana U. Math J. 53 (2004) 557-582. MR2060045 (2005f:35033)

[9] C.L. Lin and J.N. Wang, Strong unique continuation for the Lamé system with Lipschitz coefficients, Math. Ann. 331 (2005), 611-629. MR2122542

[10] G. Nakamura and J.N. Wang, Unique continuation for an elasticity system with residual stress and its applications, SIAM J. Math. Anal. 35 (2003), 304-317. MR2001103 (2004k:35037)

[11] N. Weck, Auenraumaufgaben in der Theorie stationarer Schwingungen inhomogener elasticher Korper, Math. Z. 111 (1969), 387-398. MR0263295(41:7900)

[12] _ Unique continuation for systems with Lamé principal part, Math. Methods Appl. Sci. 24 (2001), 595-605. MR1834916 (2002f:35048)

Departamento de Matematicas, Universidad del País Vasco / Euskal Herriko Unibertsitatea, Apartado 644, 48080 Bilbao, Spain

E-mail address: mtpeszul@lg.ehu.es 